\title{
Efeito Alelopático de Plantas Daninhas e Concentrações de CAPIM-BRAQUIÁRIA (Brachiaria decumbens) NO DESENVOLVIMENTO INICIAL DE EUCALIPTO (Eucalyptus grandis) ${ }^{1}$
}

\author{
Allelopathic Effect of Weeds and Concentrations of Brachiaria decumbens on the Initial \\ Development of Eucalyptus (Eucalyptus grandis)
}

SOUZA, L.S. ${ }^{2}$, VELINI, E.D. ${ }^{3}$ e MAIOMONI-RODELLA, R.C.S. ${ }^{4}$

\begin{abstract}
RESUMO - Com o objetivo de verificar possível efeito alelopático de 18 espécies de plantas daninhas sobre o crescimento inicial de Eucalyptus grandis, realizaram-se dois experimentos em casa de vegetação. No primeiro experimento, dentre 18 espécies de plantas daninhas testadas, Brachiaria decumbens (BRADC) demonstrou acentuada capacidade de reduzir o crescimento de Eucalyptus grandis, quando incorporada ao solo. No segundo experimento avaliou-se o efeito da adição no substrato de concentrações de matéria seca de BRADC sobre o crescimento inicial de mudas de E. grandis. A matéria seca triturada de BRADC foi incorporada ao solo nas concentrações de $0 \%$ (testemunha), 0,5\%, 1,0\%, 2,0\% e 3,0\% (p/p). Instalou-se também uma réplica do experimento, com adição de idênticas quantidades totais de carbono entre os tratamentos. O ajuste da quantidade de carbono foi feito através da adição de Sphagnum. Extraiu-se a solução do solo por meio de cápsulas de porcelana introduzidas nos vasos quando do transplante das mudas de eucalipto. As soluções foram coletadas ao final do experimento (39 dias após o transplante das mudas), sendo utilizadas para a determinação de $\mathrm{pH}$, condutividade elétrica, potencial osmótico e para análise de teores de nutrientes. As mínimas proporções de BRADC, com efeito inibitório, foram de 0,5 e 1,0\%, com e sem Sphagnum, respectivamente. Nestas duas condições, a área foliar média do eucalipto foi reduzida em 24 e 23\%, respectivamente. As análises de solução do solo dos tratamentos permitiram concluir que os teores de nutrientes e as características químicas destes foram pouco alterados pelos tratamentos, sendo pouco provável que a redução do crescimento do E. grandis se deva às restrições nas quantidades de nutrientes disponiveis.
\end{abstract}

Palavras-chave: alelopatia, decomposição, solução de solo, floresta e espécies daninhas.

ABSTRACT - Two experiments were carried out under greenhouse conditions to evaluate the possible allelopathic effect of 18 weed species on the initial development of eucalyptus (Eucaliptus grandis). In the first experiment, Bracharia decumbens (BRADC) caused the strongest effects mainly on the development of the upper part of the plants. In the second experiment, BRADC dry matter was added to the soil to evaluate the effect on the initial development of $\boldsymbol{E}$. grandis seedlings. The dry matter concentrations evaluated were: $O \%$ (check), $0.5 \%, 1 \%, 2 \%, 3 \% \mathrm{w} / \mathrm{w}$, incorporated into the soil. The experiment was replicated, by adding identical total quantities of carbon to the treatments. The amount of carbon was adjusted by adding Sphagnum. Soil solution was extracted through porous porcelain capsules placed in the pots (39 days after $\boldsymbol{E}$. grandis seedling transplantation). The following analyses of soil solutions collected at the end of the tests were performed: $\mathrm{pH}$ and electric conductivity, osmotic potential, and

1 Recebido para publicação em 15.5.2002 e na forma revisada em 12.12.2003.

Dissertação de mestrado do primeiro autor apresentada à FCA/UNESP-Botucatu-SP.

2 Eng.-Agr., Dr., Professor do Dep. de Fitotecnia, FCA/UNIMAR. Av. Higyno Muzzi Filho, 1001, 17525-902 Marília-SP, $<$ souza-ca@ unimar.br>; ${ }^{3}$ Eng.-Agr., Prof. Dr., Dep. de Produção Vegetal, FCA/UNESP Botucatu, Caixa Postal 237, $18603-970$ Botucatu-SP. ${ }^{4}$ Porf. Dr., IB/UNESP, Botucatu-SP. 
nutrient level analysis. Soil solution analysis of the treatments allowed to conclude that the nutrient level and the chemical characteristics of the solutions were slightly altered by the treatments, being unlikely that the reduction of $\mathbf{E}$. grandis is due to restrictions on the quantities of the available nutrients.

Key words: allelopathy, decomposition, soil solution, forest, weeds species.

\section{INTRODUÇÃO}

Muitos dos relatos de efeitos de plantas daninhas sobre as culturas têm sido classificados como "competição". Entretanto, as evidências não foram, em geral, suficientes para indicar se os efeitos foram de competição, alelopatia ou ambos (Rice, 1974). O que diferencia a alelopatia da competição entre plantas é o fato de esta competição reduzir ou remover do ambiente um fator de crescimento necessário a ambas às plantas (luz, água, nutrientes, etc.), enquanto a alelopatia ocorre pela adição de um fator ao meio. Na prática, não é fácil distinguir se o efeito nocivo de uma planta sobre a outra cabe à alelopatia ou à competição (Fuerst \& Putnan, 1983).

Efeitos alelopáticos de diversas espécies de plantas daninhas sobre diferentes culturas foram evidenciados pela pesquisa. Bhowmik \& Doll (1982, 1984) observaram que o extrato aquoso de resíduo seco de Amaranthus retroflexus inibiu o comprimento de radicelas de milho e do hipocótilo em soja. Stevens \& Tang (1987) demonstraram que Bidens pilosa reduziu o crescimento das plântulas de alface, feijão, milho e sorgo.

Devido à alta atividade biológica das Convolvulaceas, em particular das plantas do gênero Ipomoea, muitos aleloquímicos foram isolados, como alcalóides, esteróides, terpenos, fenóis e glicosídeos, entre outros (Dubey et al., 1982). Anaya et al. (1990) relatam o potencial alelopático de Ipomoea tricolor, usada na agricultura tradicional para controle de plantas daninhas. Esse potencial foi demonstrado através de medidas da atividade inibitória de lixiviados aquosos e extratos de material orgânico das plantas sobre as sementes de Amaranthus leucocarpus e Echinochloa crus-galli.

Vários trabalhos relatam a influência alelopática de Cyperus esculentus sobre as plantas cultivadas (Drost \& Doll, 1980; Drost et al., 1980). Embora as substâncias alelopáticas tenham sido comumente encontradas em extratos e resíduos das plantas, algumas foram encontradas em exsudatos de plantas vivas e gases voláteis liberados através de folhas e rizomas.

Alsaadawi et al. (1990) relataram, em observações a campo, que Euphorbia prostrata interferiu fortemente sobre Cynodon dactylon. Análises de fatores físicos e químicos do solo indicaram que a competição não foi dominante como um fator de interferência. O solo coletado na área de $E$. prostrata foi inibitório para a germinação de sementes e para o desenvolvimento das sementes de algumas espécies-teste, incluindo Cynodon dactylon. Isso sugere a presença de compostos inibitórios no solo da área de E. prostrata. Experimentos subseqüentes mostraram que extratos aquosos, resíduos decompostos e exsudatos de raízes de E. prostrata foram inibitórios para muitas plantas-teste, incluindo Cynodon dactylon.

Ito et al. (1987) avaliaram o potencial alelopático de Digitaria adscendens. Um prévio efeito inibitório foi detectado no solo sobre a emergência e o crescimento das plantas daninhas. A matéria fresca da cultura de pepino e a nodulação na soja foram menores em solo com D. adscendens. Esses resultados parecem indicar a ocorrência de alelopatia. Chou (1989) estudou a fitotoxicidade de quatro gramineas subtropicais: Brachiaria mutica, Digitaria decumbens, Imperata cylindrica e Panicum repens. O desenvolvimento de Brachiaria mutica foi inibido por Digitaria decumbens e também houve autoxicidade.

Na região de Botucatu, no Estado de São Paulo, foi constatada uma redução no desenvolvimento inicial de mudas de eucalipto (E. grandis) plantadas em antigas pastagens de capim-braquiária (B. decumbens). Com base 
nessas informações, pode-se especular a ocorrência de possíveis efeitos alelopáticos das plantas daninhas.

O presente estudo teve por objetivo avaliar os possíveis efeitos alelopáticos de 18 plantas daninhas sobre o crescimento inicial de mudas de eucalipto (Eucalyptus grandis), a fim de determinar o efeito de diferentes concentrações de matéria seca e quantificar a redução no desenvolvimento das mudas.

\section{MATERIAL E MÉTODOS}

Foram conduzidos dois experimentos em casa de vegetação, na Faculdade de Ciências Agronômicas, campus de Botucatu - UNESP, localizada na Fazenda Experimental Lageado, no município de Botucatu, Estado de São Paulo - Brasil.

O solo utilizado nos experimentos foi coletado da camada arável de um Latossolo Roxo, ocorrente na Fazenda Lageado. A terra, depois de coletada, foi seca à sombra e peneirada em peneira com malha de cinco milímetros. As principais características químicas do solo estão apresentadas na Tabela 1.

Foram utilizadas mudas da espécie Eucalyptus grandis em ambos os experimentos. As espécies de plantas daninhas estudadas foram: Ageratum conyzoides, Amaranthus lividus, Bidens pilosa, Brachiaria decumbens, Brachiaria plantaginea, Cassia occidentalis, Commelina nudiflora, Cyperus rotundus, Digitaria horizontalis, Euphorbia heterophylla, Indigofera truxillensis, Ipomoea acuminata, Panicum maximum, Peschiera fuchsiaefolia, Raphanus raphanistrum, Rhynchelytrum repens, Richardia brasiliensis e Sida cordifolia. Essas espécies foram coletadas dentro e nas proximidades da Fazenda Experimental Lageado. A parte aérea de cada uma delas foi seca em estufa de circulação forçada de ar, a
$60{ }^{\circ} \mathrm{C}$, até peso constante e, a seguir, triturada em moinho e armazenada em câmara seca.

A condução do primeiro experimento foi realizada em substrato adubado, para o crescimento das mudas, por vaso, em quantidades de nutrientes suficientes para elevar os teores de $\mathrm{P}$ a 200 ppm (Yoorim Bz), de K a 120 ppm (KCl) e de $\mathrm{N}$ a 60 ppm (sulfato de amônio). A adição dos nutrientes e a matéria seca das plantas daninhas no substrato foram feitas utilizando-se um misturador giratório manual. A parte aérea de cada planta daninha foi incorporada ao substrato, na proporção de 3\% peso/peso (p/p), constituindo-se 18 tratamentos e uma testemunha. Cada vaso continha $5 \mathrm{~kg}$ de terra como substrato. Para evitar perdas de água por percolação, foram utilizados vasos sem drenos.

Em seguida, realizou-se o transplante das mudas de eucalipto para os vasos. Ao longo do experimento, a irrigação foi efetuada de forma a manter a umidade próxima a $70 \%$ da capacidade de campo, controlando-se a necessidade de irrigação através de pesagem dos vasos, feita a cada dois dias. Após o plantio, foi colocada em cada vaso uma camada superficial de $2 \mathrm{~cm}$ de areia, para evitar o aparecimento de fungos, em decorrência da incorporação da matéria seca de plantas daninhas ao substrato. A temperatura da casa de vegetação foi mantida entre 25 e $35{ }^{\circ} \mathrm{C}$ no período diurno e entre 16 e $20^{\circ} \mathrm{C}$ no período noturno.

Ao final do experimento, 35 dias após o transplante (DAT), procedeu-se ao corte da parte aérea das mudas, separando-se as folhas e o caule (mais ramos) e medindo em seguida a área foliar com um medidor de área foliar automático, marca Hayashi Denkoh, modelo AAM-7. Os teores de clorofila foram avaliados em todas as folhas de eucalipto com equipamento de marca Minolta, modelo SPAD 502. Depois disso, os vasos foram mergulhados em

Tabela 1 - Características físico-químicas do solo dos substratos em ambos os experimentos. Botucatu-SP, 1992

\begin{tabular}{|c|c|c|c|c|c|c|c|c|c|}
\hline M.O. & $\mathrm{pH}$ & $\mathrm{P}$ & $\mathrm{K}^{+}$ & $\mathrm{Ca}^{++}$ & $\mathrm{Mg}^{++}$ & $\left(\mathrm{H}^{++} \mathrm{Al}^{+3}\right)$ & $\mathrm{S}$ & $\mathrm{CTC}$ & $\mathrm{V}(\%)$ \\
\hline$(\%)$ & $\left(\mathrm{CaCl}^{2}\right)$ & $(\mathrm{mgP} \mathrm{dm}$ & \multicolumn{7}{|c|}{$\left(\mathrm{meg} 100 \mathrm{~cm}^{-3}\right)$} \\
\hline 17,0 & 4,7 & $3,0^{\frac{1}{\prime}}$ & 0,02 & 1,4 & 0,3 & 4,2 & 1,72 & 5,92 & 29 \\
\hline
\end{tabular}

${ }^{1 /}$ método da resina (Raij \& Quaggio, 1983). 
água, procedendo-se à lavagem do substrato para a retirada das raízes. As folhas, os caules e as raízes separados foram secos em estufa de circulação forçada de ar até peso constante e sua matéria seca foi avaliada.

Foram avaliados os teores de carbono e de macro e micronutrientes presentes na matéria seca e a relação $\mathrm{C} / \mathrm{N}$ das 18 espécies de plantas daninhas apresentadas nas Tabelas 2 e 3 . A análise de nitrogênio foi efetuada segundo metodologia do semimicro Kjeldahl. Para o fósforo, utilizou-se o método do ácido fosfovanadato molíbdico (Sarruge \& Haag, 1974). Na determinação do potássio, cálcio e magnésio foi utilizado o método de espectrofotometria de absorção atômica. Para os micronutrientes cobre, zinco, ferro e manganês utilizou-se a análise de absorção atômica e, na análise do teor de carbono, o método para determinação da matéria orgânica em solo.

O delineamento experimental foi o inteiramente casualizado, com 19 tratamentos e quatro repetições.

O segundo experimento foi instalado com o objetivo de investigar o efeito de proporções crescentes de matéria seca de BRADC adicionada ao substrato sobre o crescimento inicial

Tabela 2 - Valores dos macronutrientes da matéria seca da parte aérea das plantas daninhas. Botucatu, 1992

\begin{tabular}{|c|c|c|c|c|c|c|}
\hline \multirow{2}{*}{ Espécie } & $\mathrm{N}$ & $\mathrm{P}$ & K & $\mathrm{Ca}$ & $\mathrm{Mg}$ & $S$ \\
\hline & $(\%)$ & \multicolumn{5}{|c|}{$\left(\mathrm{g} \mathrm{kg}^{-1}\right)$} \\
\hline A. conyzoides & 1,40 & 0,21 & 1,82 & 0,76 & 0,69 & 0,15 \\
\hline A.. lividus & 2,59 & 0,22 & - & 0,21 & 0,22 & 0,20 \\
\hline B. decumbens & 1,33 & 0,16 & 1,17 & 0,42 & 0,48 & 0,19 \\
\hline B. pilosa & 2,52 & 0,47 & 2,34 & 0,93 & 0,75 & 0,11 \\
\hline B. plantaginea & 1,47 & 0,13 & 1,65 & 0,27 & 0,57 & 0,19 \\
\hline C. benghalensis & 2,10 & 0,23 & 1,83 & 1,16 & 0,72 & 0,29 \\
\hline C. rotundus & 2,45 & 0,30 & 1,90 & 0,39 & 0,40 & 0,30 \\
\hline D. horizontalis & 1,82 & 0,13 & 2,18 & 0,28 & 0,50 & 0,16 \\
\hline E. heterophylla & 2,38 & 0,70 & 2,20 & 0,81 & 0,52 & 0,19 \\
\hline I. acuminata & 2,17 & 0,28 & 2,41 & 1,23 & 0,46 & 0,11 \\
\hline I. truxilensis & 2,73 & 0,14 & 0,98 & 1,16 & 0,39 & 0,08 \\
\hline P. maximum & 2,03 & 0,11 & 2,00 & 0,46 & 0,38 & 0,13 \\
\hline R. brasiliensis & 2,45 & 0,18 & 2,19 & 2,64 & 0,82 & 0,19 \\
\hline R. raphanistrum & 2,80 & 0,22 & 1,90 & 1,32 & 0,60 & 0,57 \\
\hline R. repens & 1,40 & 0,19 & 1,14 & 0,21 & 0,34 & 0,16 \\
\hline S. cordifolia & 2,31 & 0,15 & 4,02 & 3,06 & 1,17 & 0,12 \\
\hline S. occidentalis & 2,66 & 0,31 & 1,53 & 0,93 & 0,44 & 0,15 \\
\hline
\end{tabular}

de mudas de Eucalyptus grandis. Foram utilizados vasos contendo quatro litros de substrato de procedência, adubação e preparo idênticos aos do primeiro experimento. Em seguida, a matéria seca de BRADC foi incorporada ao substrato, previamente preparado, nas proporções de 0,0, 0,5, 1,0, 2,0 e 3,0\% p/p, constituindo cinco tratamentos. Outros quatro tratamentos foram montados, com a finalidade de manter constantes os teores de carbono presentes na matéria seca adicionada ao solo, procedendo-se à adição complementar de quantidades suficientes de matéria seca de Sphagnum sp. Os teores de carbono de BRADC e Sphagnum sp. foram de 43 e $32 \%$, respectivamente. Os tratamentos e as respectivas concentrações, em porcentagem, estão apresentados na Tabela 4.

A adição de Sphagnum sp. visou igualar os teores de carbono, nos tratamentos 5 a 9, decorrentes da adição de matéria orgânica ao solo. O Sphagnum sp. é comumente utilizado em experimentação como matéria orgânica inerte, quando se faz necessário (Show \& Einhellig, 1982). Em cada vaso foi ainda instalado um coletor de solução de solo com cápsulas porosas (Moraes \& Dynia, 1990). Para retirar a solução de solo acumulada nos

Tabela 3 - Valores de micronutrientes, carbono e a relação $\mathrm{C} / \mathrm{N}$ das plantas daninhas. Botucatu, 1992

\begin{tabular}{|c|c|c|c|c|c|c|}
\hline \multirow{2}{*}{ Espécie } & $\mathrm{Cu}$ & $\mathrm{Zn}$ & $\mathrm{Fe}$ & $\mathrm{Mn}$ & \multirow{2}{*}{$\frac{\mathrm{C}}{(\%)}$} & \multirow{2}{*}{$\mathrm{C} / \mathrm{N}$} \\
\hline & \multicolumn{4}{|c|}{$\left(\mathrm{mg} \mathrm{kg}^{-1}\right)$} & & \\
\hline A conyzoides & 26 & 56 & 1.666 & 18 & 40,70 & 29,07 \\
\hline A. lividus & 12 & 156 & 920 & 34 & 34,30 & 13,24 \\
\hline B. decumbens & 16 & 64 & 762 & 132 & 43,30 & 32,55 \\
\hline B. pilosa & 18 & 44 & 684 & 34 & 36,90 & 14,64 \\
\hline B. plantaginea & 14 & 48 & 800 & 44 & 41,60 & 28,30 \\
\hline C. benghalensis & 16 & 68 & 1.240 & 238 & 38,70 & 18,43 \\
\hline C. rotundus & 22 & 50 & 3.416 & 90 & 45,90 & 18,73 \\
\hline D. horizontalis & 16 & 76 & 1.306 & 152 & 40,70 & 22,36 \\
\hline E. heterophylla & 20 & 60 & 1.082 & 54 & 37,20 & 15,63 \\
\hline I. acuminata & 16 & 70 & 840 & 22 & 42,70 & 19,67 \\
\hline 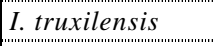 & 16 & 44 & 676 & 50 & 42,40 & 15,53 \\
\hline P. maximum & 14 & 66 & 720 & 128 & 45,60 & 22,46 \\
\hline$R$. brasiliensis & 24 & 106 & 2.978 & 122 & 37,80 & 15,42 \\
\hline R. raphanistrum & 12 & 52 & 1.304 & 186 & 37,20 & 13,28 \\
\hline R. repens & 16 & 78 & 1.070 & 166 & 47,70 & 34,07 \\
\hline S. cordifolia & 16 & 86 & 766 & 52 & 51,40 & 22,25 \\
\hline S. occidentalis & 16 & 106 & 732 & 18 & 38,40 & 14,43 \\
\hline
\end{tabular}


Tabela 4 - Porcentagens de B. decumbens e Sphagnum sp. incorporadas ao substrato e os respectivos teores de carbono que constituíram cada tratamento

\begin{tabular}{|c|c|c|c|}
\hline \multirow{2}{*}{ Tratamento } & B. decumbens & Sphagnum & Conc. Carbono \\
\cline { 2 - 4 } & \multicolumn{3}{|c|}{$(\%)$} \\
\hline 1 & 0,0 & 0,0 & 0,00 \\
\hline 2 & 0,5 & 0,0 & 0,216 \\
\hline 3 & 1,0 & 0,0 & 0,433 \\
\hline 4 & 2,0 & 0,0 & 0,866 \\
\hline 5 & 3,0 & 0,0 & 1,299 \\
\hline 6 & 0,0 & 4,15 & 1,299 \\
\hline 7 & 0,5 & 3,46 & 1,299 \\
\hline 8 & 1,0 & 2,77 & 1,299 \\
\hline 9 & 2,0 & 1,38 & 1,299 \\
\hline
\end{tabular}

coletores, ao longo do experimento, utilizou-se uma bomba de vácuo manual acoplada a uma das mangueiras (Hossner \& Phillips, 1973). As demais condições de condução do segundo experimento foram idênticas às do primeiro.

O crescimento das mudas foi acompanhado até 39 dias após o transplante (DAT), e as avaliações foram efetuadas de forma igual à relatada para o primeiro ensaio. A matéria seca de Sphagnum foi analisada quanto aos teores de nutrientes, sendo os resultados apresentados na Tabela 5.

As parcelas experimentais foram dispostas em delineamento inteiramente casualizado, num esquema fatorial $5 \times 2$, ou seja, cinco concentrações de BRADC no substrato $(0,0$, $0,5,1,0,2,0$, e 3,0\%) e duas condições, com e sem Sphagnum sp., com três repetições por tratamento.

Nos dois dias anteriores ao encerramento do experimento, foi realizada a coleta da solução de solo de todos os vasos, por meio de uma bomba a vácuo manual. As soluções assim obtidas foram recolhidas em garrafas de vidro, hermeticamente fechadas e armazenadas em geladeira, para posteriormente serem analisadas quanto ao potencial osmótico, utilizandose um osmômetro com leitura em mmol kg-1, sendo depois convertidas para pressão osmótica (-Bar). O pH foi medido com peagômetro; para a condutividade elétrica foi utilizado condutivímetro; e para os teores de nutrientes utilizou-se a mesma metodologia para a matéria seca das plantas daninhas e Sphagnum sp.

Os resultados obtidos foram submetidos à análise de variância pelo teste F. Para ambos os experimentos, procedeu-se à comparação dos tratamentos com as testemunhas através de contrastes de médias. Em vez de comparar os valores de $\mathrm{F}$ obtidos com os valores tabelados aos niveis de 5\% de probabilidade, optou-se pelo nível de significância, o qual indica a probabilidade de erro, em porcentagem, quando se admite que as duas condições são diferentes.

\section{RESULTADOS E DISCUSSÃO}

Na Tabela 6 estão apresentados os resultados dos dados referentes às mudas de eucalipto, que responderam à adição de matéria seca das 18 espécies de plantas daninhas ao solo, com redução na altura, na área foliar e no teor de clorofila aos 35 DAT. Exceto para B. pilosa, C. nudiflora, C. rotundus, E. heterophylla, I. truxilensis, P. maximum, $P$. fushsiaefolia e $R$. brasiliensis, no teor de clorofila, todas as reduções nas mudas foram significativas. Nos tratamentos com $A$. conyzoides, B. decumbens e $R$. repens, o crescimento em altura praticamente foi paralisado. Em outros tratamentos, como C. occidentalis, C. rotundus, I. truxilensis e I. acuminata, as mudas voltaram a se desenvolver. Os resultados da área foliar (Tabela 6) e da matéria seca de raiz, caule e folhas (Tabela 7), no entanto, demonstraram reduções drásticas, altamente significativas, em todos os tratamentos, refletindo o efeito inibitório por

Tabela 5 - Resultados dos teores de nutrientes da matéria seca de Sphagnum sp. Botucatu-SP, 1992

\begin{tabular}{|c|c|c|c|c|c|c|c|c|c|c|c|}
\hline $\mathrm{N}$ & $\mathrm{P}$ & $\mathrm{K}$ & $\mathrm{Ca}$ & $\mathrm{Mg}$ & $\mathrm{C}$ & $\mathrm{S}$ & $\mathrm{Cu}$ & $\mathrm{Zn}$ & $\mathrm{Fe}$ & $\mathrm{Mn}$ \\
\hline 0,93 & 0,11 & 0,53 & 2,64 & 0,10 & 32,16 & 0,06 & 06 & 20 & 900 & 50 \\
\hline
\end{tabular}


Tabela 6 - Valores médios de altura, área foliar e teor de clorofila das plantas de eucalipto e os respectivos níveis de significância para os contrastes entre os tratamentos e a testemunha. Botucatu-SP, 1992

\begin{tabular}{|c|c|c|c|c|c|c|c|c|c|}
\hline \multirow{2}{*}{ Tratamento } & \multicolumn{3}{|c|}{ Altura } & \multicolumn{3}{|c|}{ Área foliar } & \multicolumn{3}{|c|}{ Teor de clorofila } \\
\hline & $\mathrm{cm}$ & $\mathrm{F}$ & $\operatorname{Pr}>\mathrm{F}^{1 /}$ & $\mathrm{cm}^{2}$ & $\mathrm{~F}$ & $\operatorname{Pr}>F^{1 /}$ & $\mathrm{mg} \mathrm{dm}^{-2}$ & $\mathrm{~F}$ & $\operatorname{Pr}>F^{1 /}$ \\
\hline Ageratum conyzoides & 23,72 & $41,64 *$ & 0,01 & 148,46 & $121,88^{*}$ & 0,01 & 4,96 & 2,65 & 10,94 \\
\hline Amarathus lividus & 27,97 & $10,75 *$ & 0,18 & 473,80 & $28,76^{*}$ & 0,01 & 4,67 & $6,84 *$ & 1,15 \\
\hline Bidens pilosa & 25,42 & $27,94 *$ & 0,01 & 279,70 & $79,62 *$ & 0,01 & 5,02 & 1,85 & 17,91 \\
\hline Brachiaria decumbens & 21,25 & $66,46^{*}$ & 0,01 & 68,83 & $151,88^{*}$ & 0,01 & 3,24 & $78,39^{*}$ & 0,01 \\
\hline Brachiaria plantaginea & 29,62 & $5,77 *$ & 1,97 & 395,79 & $49,71 *$ & 0,01 & 4,99 & 2,28 & 13,67 \\
\hline Cassia occidentalis & 30,85 & $2,44 *$ & 2,41 & 449,66 & $38,21^{*}$ & 0,01 & 5,31 & 0,03 & 86,45 \\
\hline Commelina nudiflora & 29,50 & $6,19 *$ & 1,59 & 280,66 & $79,35^{*}$ & 0,01 & 4,65 & $8,61^{*}$ & 0,49 \\
\hline Cyperus rotundus & 30,87 & $2,39 *$ & 2,82 & 661,76 & $7,62 *$ & 0,78 & 5,35 & 0,00 & 97,58 \\
\hline Digitaria horizontalis & 29,00 & $8,02 *$ & 0,65 & 285,22 & $78,04^{*}$ & 0,01 & 5,00 & 2,18 & 14,52 \\
\hline Euphorbia heterophylla & 25,25 & $29,23^{*}$ & 0,01 & 222,66 & $96,88^{*}$ & 0,01 & 5,15 & 0,68 & 41,37 \\
\hline Indigofera truxilensis & 31,75 & $0,89 *$ & 4,93 & 352,66 & $60,01^{*}$ & 0,01 & 5,15 & 0,68 & 41,32 \\
\hline Ipomoea acuminata & 32,0 & $0,60 *$ & 4,32 & 344,09 & $62,17^{*}$ & 0,01 & 4,78 & $5,75^{*}$ & 1,99 \\
\hline Panicum maximum & 29,35 & $6,72 *$ & 1,22 & 478,31 & $32,72^{*}$ & 0,01 & 5,08 & 1,22 & 27,47 \\
\hline Peschiera fuschsiaefolia & 29,50 & $6,19 *$ & 1,59 & 471,14 & $34,05^{*}$ & 0,01 & 5,51 & 0,48 & 48,92 \\
\hline Raphanus raphanistrum & 30,50 & $3,25^{*}$ & 7,70 & 401,17 & $48,50^{*}$ & 0,01 & 4,97 & 2,51 & 11,89 \\
\hline Rhynchelytum repens & 23,90 & $34,38 *$ & 0,01 & 199,20 & $89,55^{*}$ & 0,01 & 4,37 & $14,3 * 8$ & 0,04 \\
\hline Richardia brasiliensis & 30,45 & $3,37^{*}$ & 7,17 & 446,06 & $38,94^{*}$ & 0,01 & 5,14 & 0,75 & 38,93 \\
\hline Sida cordifolia & 30,05 & $4,46 *$ & 3,93 & 428,14 & $42,63^{*}$ & 0,01 & 4,67 & $8,11^{*}$ & 0,62 \\
\hline Testemunha & 33,12 & - & - & 832,95 & & & 5,35 & & \\
\hline $\mathrm{F}$ tratamento & - & 9,45 & 0,01 & & 15,95 & 0,01 & & 8,49 & 0,01 \\
\hline $\mathrm{CV}(\%)$ & \multicolumn{3}{|c|}{7,17} & \multicolumn{3}{|c|}{23,00} & \multicolumn{3}{|c|}{6,81} \\
\hline
\end{tabular}

${ }^{1 /}$ Probabilidade com significância em porcentagem de 0,01 a 100; as médias com * são significativas a 10\% de probabilidade.

parte de todas as plantas daninhas testadas. A redução na acumulação de matéria seca na planta pode estar relacionada com a redução na área foliar e no teor de clorofila. Sendo as folhas responsáveis pela interceptação de luz e captação de $\mathrm{CO}_{2}$ e local primário da fotossíntese, a redução na área foliar e no teor de clorofila desfavorece o crescimento do eucalipto, levando à redução na matéria seca total. Conforme ressaltaram Blum et al. (1987), a área foliar é um parâmetro importante a ser estudado em trabalhos que envolvem o efeito de compostos aleloquímicos, uma vez que há correlação positiva entre área foliar e matéria seca da planta.

Relatos sobre a ocorrência de formas de ação alelopática podem ser encontrados para diversas plantas daninhas utilizadas no presente trabalho, ou espécies apresentadas, como, por exemplo, Bidens pilosa (Stevens \& Tang, 1987), Ipomoea spp. (Anaya et al., 1990; Labonte \& Darding, 1988), Cyperus rotundus
(Labrada et al., 1986), Euphorbia prostrata (Alsaadawi et al., 1990), Digitaria adscendes (Ito et al., 1987), Raphanus sativa, Sida acuta, Sida rhombifolia, Panicum fasciculatum e Euphorbia sp. (Gliessman, 1983), Digitaria decumbens, Panicum repens e Panicum maximum (Chou, 1987).

Analisando os resultados em conjunto, nota-se que, de modo geral, todos os tratamentos produziram reduções nos parâmetros investigados, havendo, contudo, efeitos mais drásticos em alguns casos. Destes, podem-se destacar BRADC e A. conyzoides, em ordem decrescente, como as plantas daninhas que provocaram efeitos mais drásticos na redução do crescimento das mudas de eucalipto, com base nos parâmetros analisados. Estudos de alelopatia relatam a utilização de substâncias orgânicas, decompostas no solo, a partir de plantas secas (Rice, 1974; Wilson \& Rice, 968). Nesse sentido, alguns estudos demonstram que determinados fungos, que agem na decomposição do 
material incorporado ao solo, originam produtos que interagem no metabolismo da planta (Norstat \& Mccalla, 1963). Do ponto de vista de Rice (1974), em estudos feitos com restos de plantas no solo, é difícil determinar qual o agente tóxico envolvido - se um microrganismo ou se resultado de um aditivo ou um efeito sinergístico.

Estudos de compostos aleloquímicos, adicicionados ao solo, sugerem que vários fatores, como argila, óxidos, matéria orgânica, pH, nutrientes e microrganismos, determinam as concentrações ativas desses compostos nos solos (Dalton et al., 1983; Wang et al., 1978).

Em observações realizadas a campo, o capim-braquiária (B. decumbens) demonstrou ser uma planta daninha prejudicial, quando incorporada ao solo, nas áreas de implantação de mudas de eucalipto. Pelo fato de a BRADC ter apresentado evidências inibitórias altamente significativas no primeiro ensaio, esta planta foi utilizada no segundo ensaio.
Para minimizar o efeito da relação $\mathrm{C} / \mathrm{N}$ na decomposição da matéria seca, utilizou-se o Sphagnum sp., material inerte (Shcow \& Einhellig, 1982) como fonte de carbono, incorporado nas diferentes proporções de $B$. decumbens, pois sabe-se que, durante a decomposição microbiana e degradação de enzimas das partes da planta, uma enorme variedade de elementos químicos são formados e dissolvidos no solo, onde muitos desempenham importante atividade biológica. Substâncias alelopáticas aparecem nesse tipo de decomposição atuando na respiração, na germinação e no desenvolvimento, em muitas áreas agrícolas (Chou \& Lin, 1976; Chou \& Kuo, 1986). Os tratamentos com Sphagnum sp. tiveram como objetivo manter a concentração de carbono constante nas diferentes proporções de BRADC, a fim de minimizar o efeito $\mathrm{C} / \mathrm{N}$. Nas Tabelas 8 e 9 estão apresentados os valores médios de altura, área foliar, teor de clorofila, quantidade de clorofila total e da massa seca de folhas, caules, raiz e total das plantas de

Tabela 7 - Valores de massa seca das folhas, do caule e da raiz das plantas de eucalipto e os respectivos níveis de significância para os contrastes entre os tratamentos e a testemunha. Botucatu-SP, 1992

\begin{tabular}{|c|c|c|c|c|c|c|c|c|c|}
\hline \multirow{2}{*}{ Tratamento } & \multicolumn{9}{|c|}{ Massa seca (g) } \\
\hline & Folhas & $\mathrm{F}$ & $\operatorname{Pr}>F^{1 /}$ & Caule & $\mathrm{F}$ & $\operatorname{Pr}>\mathrm{F}^{1 /}$ & Raiz & $\mathrm{F}$ & $\operatorname{Pr}>F^{1 /}$ \\
\hline Ageratum conyzoides & 0,610 & $146,23^{*}$ & 0,01 & 0,285 & $136,70 *$ & 0,01 & 0,522 & $26,99 *$ & 0,01 \\
\hline Amarathus lividus & 1,626 & $33,88^{*}$ & 0,01 & 0,670 & $40,09 *$ & 0,01 & 0,676 & $11,21 *$ & 0,15 \\
\hline Bidens pilosa & 1,070 & $89,60^{*}$ & 0,01 & 0,402 & $104,26^{*}$ & 0,01 & 0,732 & $9,28 *$ & 0,36 \\
\hline Brachiaria decumbens & 0,372 & $180,88^{*}$ & 0,01 & 0,212 & $158,90^{*}$ & 0,01 & 0,535 & $25,68^{*}$ & 0,01 \\
\hline Brachiaria plantaginea & 1,352 & $61,66^{*}$ & 0,01 & 0,587 & $62,07 *$ & 0,01 & 0,645 & $15,53^{*}$ & 0,02 \\
\hline Cassia occidentalis & 1,602 & $41,28^{*}$ & 0,01 & 0,645 & $51,18^{*}$ & 0,01 & 0,710 & $10,73^{*}$ & 0,18 \\
\hline Commelina nudiflora & 1,045 & $92,32 *$ & 0,01 & 0,480 & $85,26^{*}$ & 0,01 & 0,615 & $18,05^{*}$ & 0,01 \\
\hline Cyperus rotundus & 1,917 & $21,40^{*}$ & 0,01 & 0,892 & $16,27 *$ & 0,02 & 0,845 & $3,59^{*}$ & 6,35 \\
\hline Digitaria horizontalis & 1,015 & $95,65^{*}$ & 0,01 & 0,450 & $92,39 *$ & 0,01 & 0,472 & $32,57 *$ & 0,01 \\
\hline Euphorbia heterophylla & 0,867 & $112,83^{*}$ & 0,01 & 0,350 & $118,21^{*}$ & 0,01 & 0,472 & $32,57^{*}$ & 0,01 \\
\hline Indigofera truxilensis & 1,365 & $60,54^{*}$ & 0,01 & 0,567 & $66,11^{*}$ & 0,01 & 0,705 & $11,07^{*}$ & 0,16 \\
\hline Ipomoea acuminata & 1,135 & $82,71^{*}$ & 0,01 & 0,520 & $76,20^{*}$ & 0,01 & 0,537 & $25,42 *$ & 0,01 \\
\hline Panicum maximum & 1,725 & $32,78^{*}$ & 0,01 & 0,747 & $34,36^{*}$ & 0,01 & 0,810 & $5,07 *$ & 2,83 \\
\hline Peschiera fuschsiaefolia & 1,710 & $33,77^{*}$ & 0,01 & 0,750 & $33,99 *$ & 0,01 & 0,715 & $10,40^{*}$ & 0,21 \\
\hline Raphanus raphanistrum & 1,650 & $37,87^{*}$ & 0,01 & 0,705 & $40,93^{*}$ & 0,01 & 0,707 & $10,90^{*}$ & 0,17 \\
\hline Rhynchelytum repens & 0,813 & $102,43^{*}$ & 0,01 & 0,353 & $100,54 *$ & 0,01 & 0,646 & $13,20^{*}$ & 0,06 \\
\hline Richardia brasiliensis & 1,565 & $44,07 *$ & 0,01 & 0,737 & $35,85^{*}$ & 0,01 & 0,660 & $14,35^{*}$ & 0,04 \\
\hline Sida cordifolia & 1,430 & $54,91 *$ & 0,01 & 0,602 & $59,13 *$ & 0,01 & 0,512 & $28,07 *$ & 0,01 \\
\hline Testemunha & 2,727 & - & - & 1,212 & - & - & 1,030 & - & - \\
\hline $\mathrm{F}$ tratamento & - & 18,25 & 0,01 & - & 17,30 & 0,01 & - & 4,11 & 0,01 \\
\hline $\mathrm{CV}(\%)$ & \multicolumn{3}{|c|}{18,33} & \multicolumn{3}{|c|}{19,01} & \multicolumn{3}{|c|}{20,91} \\
\hline
\end{tabular}

${ }^{1 /}$ Probabilidade com significância em porcentagem de 0,01 a 100; as médias com * são significativas a 10\% de probabilidade. 
eucalipto, bem como os respectivos níveis de significância para os contrastes entre as diferentes proporções de BRADC, com e sem adição de Sphagnum sp., avaliados aos 39 DAT. De modo geral, em todos os parâmetros analisados, os quais envolvem um gradiente de proporções de BRADC com Sphagnum sp., os efeitos de redução no desenvolvimento das mudas foram significativos. O contraste entre os tratamentos T6 e T5, os quais apresentam o mesmo teor de carbono, demonstrou efeito altamente significativo em todos os parâmetros analisados. Com esse procedimento, verificou-se a nãointerferência do fator $\mathrm{C} / \mathrm{N}$, realizado no primeiro ensaio, com a proporção máxima de $3 \%(\mathrm{p} / \mathrm{p})$ de matéria seca das plantas daninhas.

Segundo Neptune (1976), a imobilização e a mineralização de nutrientes dependem dos teores de carbono e nitrogênio, como também da relação entre estes dois elementos, nos resíduos orgânicos adicionados ao solo. A relação $\mathrm{C} / \mathrm{N}$ indica claramente a disponibilidade do nitrogênio em relação ao carbono e a rapidez com a qual se processa a mineralização. Embora BRADC e B. plantaginea pertençam ao mesmo gênero e apresentem relação $\mathrm{C} / \mathrm{N}$ semelhante, esta última não apresentou o mesmo efeito inibitório observado pela BRADC nas mudas de eucalipto analisadas. Entretanto, a espécie $R$. roseum apresentou uma relação $\mathrm{C} / \mathrm{N}$ maior que BRADC, porém o seu efeito inibitório não foi tão intenso quanto o observado por esta última. A relação $\mathrm{C} / \mathrm{N}$ de $\mathrm{BRADC}$ (32) indicou que não há possibilidade de imobilização de nutrientes - sabe-se que a completa imobilização de nutriente encontrase em relações $\mathrm{C} / \mathrm{N}$ maiores que 33:1

Tabela 8 - Valores médios de altura, área foliar, teor de clorofila e quantidade de clorofila total das plantas de eucalipto e os respectivos níveis de significância para os contrastes entre as diferentes proporções de BRADC, com e sem adição de Sphagnum sp., avaliados aos 39 DAT. Botucatu-SP, 1993

\begin{tabular}{|c|c|c|c|c|c|c|c|c|}
\hline Tratamento & \multicolumn{2}{|c|}{ Altura $(\mathrm{cm})$} & \multicolumn{2}{|c|}{ Área foliar $\left(\mathrm{cm}^{2}\right)$} & \multicolumn{2}{|c|}{$\begin{array}{c}\text { Teor de clorofila } \\
\left(\mathrm{mg} \mathrm{dm}^{-2}\right)\end{array}$} & \multicolumn{2}{|c|}{$\begin{array}{l}\text { Total de clorofila } \\
\text { (mg por planta) }\end{array}$} \\
\hline $1(0,0$ BRADC $+0,00$ Sphagnum $=0,000 \% \mathrm{C})$ & \multicolumn{2}{|c|}{36,17} & \multicolumn{2}{|c|}{1178,42} & \multicolumn{2}{|c|}{5,65} & \multicolumn{2}{|c|}{67,05} \\
\hline $2(0,5$ BRADC $+0,00$ Sphagnum $=0,000 \%$ C) & \multicolumn{2}{|c|}{36,83} & \multicolumn{2}{|c|}{1318,94} & \multicolumn{2}{|c|}{5,58} & \multicolumn{2}{|c|}{73,58} \\
\hline $3(1,0$ BRADC $+0,00$ Sphagnum $=0,000 \% \mathrm{C})$ & \multicolumn{2}{|c|}{35,67} & \multicolumn{2}{|c|}{903,41} & \multicolumn{2}{|c|}{5,48} & \multicolumn{2}{|c|}{49,49} \\
\hline $4(2,0$ BRADC $+0,00$ Sphagnum $=0,000 \% \mathrm{C})$ & \multicolumn{2}{|c|}{24,93} & \multicolumn{2}{|c|}{271,04} & \multicolumn{2}{|c|}{5,13} & \multicolumn{2}{|c|}{14,03} \\
\hline $5(3,0$ BRADC $+0,00$ Sphagnum $=1,299 \% \mathrm{C})$ & \multicolumn{2}{|c|}{23,83} & \multicolumn{2}{|c|}{140,76} & \multicolumn{2}{|c|}{4,48} & \multicolumn{2}{|c|}{6,30} \\
\hline $6(0,0$ BRADC $+4,15$ Sphagnum $=1,299 \%$ C $)$ & \multicolumn{2}{|c|}{33,33} & \multicolumn{2}{|c|}{1516,93} & \multicolumn{2}{|c|}{6,02} & \multicolumn{2}{|c|}{90,06} \\
\hline $7(0,5$ BRADC $+3,46$ Sphagnum $=1,299 \% \mathrm{C})$ & \multicolumn{2}{|c|}{32,00} & \multicolumn{2}{|c|}{1147,93} & \multicolumn{2}{|c|}{5,79} & \multicolumn{2}{|c|}{65,96} \\
\hline $8(1,0$ BRADC $+2,77$ Sphagnum $=1,299 \%$ C $)$ & & & 1016 & & & 49 & & \\
\hline $9(2,0$ BRADC $+1,38$ Sphagnum $=1,299 \%$ C $)$ & & & 420 & & & 22 & & \\
\hline Contrastes entre Tratamentos & $\mathrm{F}$ & $\operatorname{Pr}>\mathrm{F}^{1 /}$ & $\mathrm{F}$ & $\operatorname{Pr}>\mathrm{F}^{1 /}$ & $\mathrm{F}$ & $\operatorname{Pr}>\mathrm{F}^{1 /}$ & $\mathrm{F}$ & $\operatorname{Pr}>F^{1 /}$ \\
\hline 1 vs. 2 & 0,10 & 76,09 & 0,99 & 33,27 & 0,05 & 82,58 & 0,79 & 38,42 \\
\hline 1 vs. 3 & 0,05 & 81,94 & $3,78^{*}$ & 6,62 & 0,29 & 59,42 & $5,72 *$ & 2,67 \\
\hline 1 vs. 4 & $27,01^{*}$ & 0,01 & $41,10^{*}$ & 0,01 & 2,74 & 11,33 & $52,18^{*}$ & 0,01 \\
\hline 1 vs. 5 & $32,56^{*}$ & 0,01 & $53,75^{*}$ & 0,01 & $14,04 *$ & 0,13 & $68,48^{*}$ & 0,01 \\
\hline 6 vs. 7 & 0,38 & 54,43 & 2,30 & 14,52 & 0,51 & 48,51 & $10,78^{*}$ & 0,37 \\
\hline 6 vs. 8 & 0,72 & 40,64 & $6,80^{*}$ & 1,69 & 2,85 & 10,70 & $21,45^{*}$ & 0,02 \\
\hline 6 vs. 9 & $12,58^{*}$ & 0,20 & $12,52^{*}$ & 0,21 & $6,44^{*}$ & 1,96 & $86,24 *$ & 0,01 \\
\hline Sem Sphagnum vs. Com Sphagnum & 2,96 & 10,10 & $60,05^{*}$ & 0,01 & 1,12 & 30,34 & $4,13^{*}$ & 5,55 \\
\hline 1 vs. 6 & 1,72 & 20,48 & $5,72 *$ & 2,67 & 1,34 & 26,08 & $9,83 *$ & 0,52 \\
\hline 2 vs. 7 & $5,00 *$ & 3,69 & 1,46 & 24,11 & 0,45 & 51,13 & 1,08 & 31,18 \\
\hline 3 vs. 8 & 0,05 & 81,94 & 0,63 & 43,52 & 0,00 & 99,16 & 0,80 & 38,09 \\
\hline 4 vs. 9 & 0,12 & 73,79 & 1,11 & 30,51 & 0,08 & 78,54 & 1,15 & 29,64 \\
\hline 6 vs. 5 & $19,32 *$ & 0,03 & $94,53^{*}$ & 0,01 & $24,06^{*}$ & 0,01 & $130,21 *$ & 0,01 \\
\hline $\mathrm{F}$ tratamentos & 12,36 & 0,01 & 22,53 & 0,01 & 3,70 & 0,71 & 30,00 & 0,01 \\
\hline $\mathrm{CV}(\%)$ & & & 20 & & & & & \\
\hline
\end{tabular}

\footnotetext{
${ }^{1 /}$ Probabilidade com significância em porcentagem de 0,01 a 100; as médias com * são significativas a 10\% de probabilidade.
} 
(Neptune, 1976). Os contrastes entre as proporções de BRADC correspondentes nos tratamentos com e sem Sphagnum (SS vs. CS), de modo geral, não foram significativos, exceto entre os tratamentos envolvendo $0,5 \%$ de BRADC (T2 vs. T7), indicando que o mínimo de proporção com efeito inibitório nos tratamentos com e sem Sphagnum proporcionou um efeito estimulante nas mudas de eucalipto. Esse efeito estimulatório pode ser devido à rápida mineralização da matéria orgânica, liberando nutrientes no solo disponível para a planta. De acordo com Alves (1992), a divisão e o alongamento celular são fases essenciais do crescimento e desenvolvimento e acreditase que os inibidores atuem principalmente nesses processos.

Os dados de teor de clorofila total (mg por planta) revelam redução significativa nos gradientes em BRADC sem Sphagnum sp. e pequena redução com Sphagnum sp. Como a clorofila total está relacionada com a matéria seca total, conseqüentemente, os dados mostram que houve inibição de fotossíntese (Putnam, 1986).

Na tentativa de verificar os possiveis efeitos causados por retenção de água, salinidade, $\mathrm{pH}$, teores de nutrientes com a adição de matéria seca, foram coletadas as soluções de solo para as análises de potencial osmótico, condutividade elétrica, $\mathrm{pH}$ e teores de nutrientes (Tabelas 10 e 11). Estudos de solos nos quais compostos alelopáticos foram adicionados sugerem o envolvimento de vários fatores (argila, óxidos, matéria orgânica, $\mathrm{pH}$, nutrientes e microrganismos), que têm papel importante na determinação de concentrações ativas de compostos nos solos (Dalton et al., 1983; Wang et al., 1978). Segundo Blum et al. (1987), a concentração ativa é definida como uma fração de compostos alelopáticos que se encontra disponível na solução de solo.

Tabela 9 - Valores médios de massa seca de folhas, caules, raiz e total das plantas de eucalipto e os respectivos níveis de significância para os contrastes entre as diferentes proporções de BRADC, com e sem adição de Sphagnum sp., avaliados aos 39 DAT. Botucatu-SP, 1993

\begin{tabular}{|c|c|c|c|c|c|c|c|c|}
\hline \multirow{2}{*}{ Tratamento } & \multicolumn{8}{|c|}{ Massa seca $(\mathrm{g})$} \\
\hline & \multicolumn{2}{|c|}{ Folhas } & \multicolumn{2}{|c|}{ Caule } & \multicolumn{2}{|c|}{ Raiz } & \multicolumn{2}{|c|}{ Total } \\
\hline $1(0,0$ BRADC $+0,00$ Sphagnum $=0,000 \% \mathrm{C})$ & \multicolumn{2}{|c|}{4,54} & \multicolumn{2}{|c|}{2,55} & \multicolumn{2}{|c|}{4,41} & \multicolumn{2}{|c|}{11,50} \\
\hline $2(0,5$ BRADC $+0,00$ Sphagnum $=0,000 \% \mathrm{C})$ & \multicolumn{2}{|c|}{5,30} & \multicolumn{2}{|c|}{2,51} & \multicolumn{2}{|c|}{4,79} & \multicolumn{2}{|c|}{12,61} \\
\hline $3(1,0$ BRADC $+0,00$ Sphagnum $=0,000 \% \mathrm{C})$ & \multicolumn{2}{|c|}{4,14} & \multicolumn{2}{|c|}{1,77} & \multicolumn{2}{|c|}{4,83} & \multicolumn{2}{|c|}{10,75} \\
\hline $4(2,0 \mathrm{BRADC}+0,00$ Sphagnum $=0,000 \% \mathrm{C})$ & \multicolumn{2}{|c|}{1,18} & \multicolumn{2}{|c|}{0,59} & \multicolumn{2}{|c|}{2,64} & \multicolumn{2}{|c|}{4,41} \\
\hline $5(3,0$ BRADC $+0,00$ Sphagnum $=1,299 \%$ C $)$ & \multicolumn{2}{|c|}{0,74} & \multicolumn{2}{|c|}{0,36} & \multicolumn{2}{|c|}{1,94} & \multicolumn{2}{|c|}{3,05} \\
\hline $6(0,0$ BRADC $+4,15$ Sphagnum $=1,299 \% \mathrm{C})$ & \multicolumn{2}{|c|}{5,49} & \multicolumn{2}{|c|}{2,39} & \multicolumn{2}{|c|}{5,32} & \multicolumn{2}{|c|}{13,20} \\
\hline $7(0,5$ BRADC $+3,46$ Sphagnum $=1,299 \%$ C $)$ & \multicolumn{2}{|c|}{4,29} & & & & & & 66 \\
\hline $8(1,0$ BRADC $+2,77$ Sphagnum $=1,299 \% \mathrm{C})$ & & & & & & & & 11 \\
\hline $9(2,0$ BRADC $+1,38$ Sphagnum $=1,299 \% \mathrm{C})$ & & & & & & & & 80 \\
\hline Contrastes entre Tratamentos & $\mathrm{F}$ & $\operatorname{Pr}>\mathrm{F}^{1 /}$ & $\mathrm{F}$ & $\operatorname{Pr}>\mathrm{F}^{1 / 1}$ & $\mathrm{~F}$ & $\operatorname{Pr}>\mathrm{F}^{1 /}$ & $\mathrm{F}$ & $\operatorname{Pr}>\mathrm{F}^{1 /}$ \\
\hline 1 vs. 2 & 2,36 & 14,05 & 0,02 & 87,75 & 0,10 & 75,25 & 0,55 & 46,73 \\
\hline 1 vs. 3 & 0,62 & 44,00 & $10,93^{*}$ & 0,35 & 0,12 & 72,98 & 0,25 & 62,31 \\
\hline 1 vs. 4 & $45,17 *$ & 0,01 & $69,62 *$ & 0,01 & 2,18 & 15,55 & $22,25^{*}$ & 0,01 \\
\hline 1 vs. 5 & $57,78^{*}$ & 0,01 & $86,39^{*}$ & 0,01 & $4,25^{*}$ & 5,24 & $31,66^{*}$ & 0,01 \\
\hline 6 vs. 7 & $5,71 *$ & 2,68 & $4,41 *$ & 4,86 & 0,51 & 48,33 & 2,87 & 10,60 \\
\hline 6 vs. 8 & $7,23 *$ & 1,41 & 2,48 & 13,09 & 1,31 & 26,56 & $4,22 *$ & 5,32 \\
\hline 6 vs. 9 & $57,99 *$ & 0,01 & $54,87 *$ & 0,01 & $4,27 *$ & 5,21 & $28,49 *$ & 0,01 \\
\hline Sem Sphagnum vs. Com Sphagnum & 0,20 & 66,19 & 1,00 & 32,91 & 0,00 & 96,72 & 0,00 & 96,68 \\
\hline 1 vs. 6 & $6,62 *$ & 7,17 & 0,46 & 50,36 & 0,58 & 45,68 & 1,28 & 27,12 \\
\hline 2 vs. 7 & $4,09^{*}$ & 5,67 & $6,89 *$ & 1,62 & 0,08 & 78,60 & 1,70 & 20,76 \\
\hline 3 vs. 8 & 0,00 & 32,56 & 1,10 & 30,62 & 0,54 & 46,99 & 0,18 & 67,63 \\
\hline 4 vs. 9 & 1,02 & 32,56 & 0,07 & 80,10 & 0,03 & 86,71 & 0,26 & 61,54 \\
\hline 6 vs. 5 & $90,32 *$ & 0,01 & $74,19^{*}$ & 0,01 & $7,79 *$ & 1,05 & $45,67 *$ & 0,01 \\
\hline F tratamentos & 24,76 & 0,01 & 27,57 & 0,01 & 2,13 & 7,61 & 12,84 & 0,01 \\
\hline $\mathrm{CV}(\%)$ & 24 & & & & & & & \\
\hline
\end{tabular}

${ }^{1 /}$ Probabilidade com significância em porcentagem de 0,01 a 100; as médias com * são significativas a $10 \%$ de probabilidade. 
Tabela 10 - Valores médios de potencial osmótico, condutividade elétrica e pH da solução de solo plantas de eucalipto e os respectivos níveis de significância para os contrastes entre as diferentes proporções de BRADC, com e sem adição de Sphagnum sp., avaliados aos 39 DAT. Botucatu-SP, 1993

\begin{tabular}{|c|c|c|c|c|c|c|}
\hline \multirow[b]{2}{*}{ Tratamento } & \multicolumn{6}{|c|}{ Solução de Solo } \\
\hline & \multicolumn{2}{|c|}{$\begin{array}{c}\text { Potencial osmótico } \\
\text { (- Bar) }\end{array}$} & \multicolumn{2}{|c|}{$\begin{array}{c}\text { Condutividade eletrica } \\
\left(\mu \mathrm{s} \mathrm{cm}^{-1}\right)\end{array}$} & \multicolumn{2}{|c|}{$\mathrm{pH}$} \\
\hline $1(0,0$ BRADC $+0,00$ Sphagnum $=0,000 \%$ C $)$ & \multicolumn{2}{|c|}{1,19} & \multicolumn{2}{|c|}{$1.317,50$} & \multicolumn{2}{|c|}{6,39} \\
\hline $2(0,5$ BRADC $+0,00$ Sphagnum $=0,000 \%$ C $)$ & \multicolumn{2}{|c|}{1,27} & \multicolumn{2}{|c|}{$1.271,66$} & \multicolumn{2}{|c|}{7,03} \\
\hline $3(1,0$ BRADC $+0,00$ Sphagnum $=0,000 \% \mathrm{C})$ & \multicolumn{2}{|c|}{1,17} & \multicolumn{2}{|c|}{$1.108,66$} & \multicolumn{2}{|c|}{7,01} \\
\hline $4(2,0$ BRADC $+0,00$ Sphagnum $=0,000 \% \mathrm{C})$ & \multicolumn{2}{|c|}{1,34} & \multicolumn{2}{|c|}{$1.928,00$} & \multicolumn{2}{|c|}{7,35} \\
\hline $5(3,0$ BRADC $+0,00$ Sphagnum $=1,299 \% \mathrm{C})$ & \multicolumn{2}{|c|}{1,43} & \multicolumn{2}{|c|}{$2.306,66$} & \multicolumn{2}{|c|}{7,43} \\
\hline $6(0,0$ BRADC $+4,15$ Sphagnum $=1,299 \% \mathrm{C})$ & \multicolumn{2}{|c|}{1,52} & \multicolumn{2}{|c|}{$3.003,33$} & \multicolumn{2}{|c|}{6,67} \\
\hline $7(0,5$ BRADC + 3,46 Sphagnum $=1,299 \%$ C) & \multicolumn{2}{|c|}{1,19} & \multicolumn{2}{|c|}{$2.008,66$} & \multicolumn{2}{|c|}{ 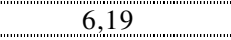 } \\
\hline $8(1,0$ BRADC $+2,77$ Sphagnum $=1,299 \%$ C $)$ & \multicolumn{2}{|c|}{1,01} & \multicolumn{2}{|c|}{$1.632,00$} & \multicolumn{2}{|c|}{6,37} \\
\hline $9(2,0$ BRADC $+1,38$ Sphagnum $=1,299 \% \mathrm{C})$ & \multicolumn{2}{|c|}{1,11} & \multicolumn{2}{|c|}{$1.609,33$} & \multicolumn{2}{|c|}{7,17} \\
\hline Contrastes entre Tratamentos & $\mathrm{F}$ & $\operatorname{Pr}>\mathrm{F}^{1 /}$ & $\mathrm{F}$ & $\operatorname{Pr}>\mathrm{F}^{1 /}$ & $\mathrm{F}$ & $\operatorname{Pr}>F^{1 /}$ \\
\hline 1 vs. 2 & 0,32 & 57,59 & 0,01 & 92,01 & 1,72 & 20,51 \\
\hline 1 vs. 3 & 0,02 & 88,84 & 0,21 & 64,86 & 1,58 & 22,37 \\
\hline 1 vs. 4 & 1,14 & 29,85 & 1,83 & 19,18 & $3,82 *$ & 6,48 \\
\hline 1 vs. 5 & 2,91 & 10,35 & $4,81 *$ & 4,10 & $4,48^{*}$ & 4,69 \\
\hline 6 vs. 7 & $5,50 *$ & 2,95 & $6,08 *$ & 2,34 & 0,93 & 34,67 \\
\hline 6 vs. 8 & $13,13^{*}$ & 0,17 & $11,55^{*}$ & 0,30 & 0,36 & 55,26 \\
\hline 6 vs. 9 & $8,47^{*}$ & 0,86 & $11,94^{*}$ & 0,27 & 1,04 & 32,08 \\
\hline Sem Sphagnum vs. Com Sphagnum & 0,25 & 62,42 & $9,98^{*}$ & 0,52 & 1,98 & 17,44 \\
\hline 1 vs. 6 & $5,50 *$ & 2,95 & $13,97 *$ & 0,14 & 0,32 & 57,95 \\
\hline 2 vs. 7 & 1,32 & 57,59 & $3,34 *$ & 8,35 & 2,93 & 10,27 \\
\hline 3 vs. 8 & 1,29 & 26,89 & 1,68 & 21,01 & 1,68 & 20,96 \\
\hline 4 vs. 9 & 2,67 & 11,80 & 0,62 & 43,94 & 0,14 & 71,29 \\
\hline 6 vs. 5 & 0,41 & 53,04 & 2,98 & 10,04 & 2,42 & 13,58 \\
\hline $\mathrm{F}$ tratamentos & 2,34 & 5,43 & 3,75 & 0,74 & 2,31 & 5,32 \\
\hline $\mathrm{CV}(\%)$ & & & & & & \\
\hline
\end{tabular}

\footnotetext{
${ }^{1 /}$ Probabilidade com significância em porcentagem de 0,01 a 100; as médias com * são significativas a 10\% de probabilidade.
}

Na Tabela 10 estão apresentados os valores médios de potencial osmótico, condutividade elétrica e pH da solução de solo e os respectivos níveis de significância para os contrastes entre as diferentes concentrações de BRADC, com e sem adição de Sphagnum sp., avaliados aos 39 DAT. A análise de potencial osmótico demonstrou que não houve retenção de água disponivel para as mudas de eucalipto. O tratamento com 3\% (p/p) de Sphagnum sp. indicou o maior índice (1,52 -Bar), longe de uma retenção de água, pois uma alta retenção indica índice abaixo de 3,00 -Bar, sendo o potencial osmótico determinado pela concentração de sais do soluto e não pela concentração de matéria orgânica no solo.

Verificou-se, através dos dados da condutividade elétrica, que a salinidade da solução de solo permaneceu em níveis moderados de salinidade (750 a 2.250 micromhos $\left.\mathrm{cm}^{-1}\right)$. De acordo com Neptune (1976), pode-se considerar que não houve efeito de acumulação de sais na solução de solo. À medida que se aumentava a proporção de $B$. decumbens no solo, diminuía o índice de condutividade elétrica na solução de solo com Sphagnum sp. (Tabela 10).

$\mathrm{O}$ pH da solução de solo manteve-se na faixa neutra em todos os tratamentos, tanto com ou sem Sphagnum sp., provavelmente em decorrência do poder-tampão do solo ou do alto teor de Ca no Sphagnum sp., funcionando como um corretivo do solo.

Os resultados das análises dos teores de nutrientes (N, K, Ca e Mg), apresentados na Tabela 11, indicaram que, à medida que se aumentava a concentração de BRADC, aumentavam os teores de N, K, Ca e Mg na solução de solo sem Sphagnum sp. Nos tratamentos com Sphagnum sp., à medida que diminuiu a proporção de Sphagnum sp., reduziram-se os teores de nutrientes. Segundo Chou (1987), durante a decomposição de resíduos de arroz no solo, a quantidade de minerais disponiveis poderá ser afetada e, conseqüentemente, modificar o crescimento da planta. 
Tabela 11 - Valores médios dos teores de nitrogênio, potássio, cálcio e magnésio da solução de solo e os respectivos níveis de significância para os contrastes entre as diferentes proporções de BRADC, com e sem adição de Sphagnum sp., avaliados aos 39 DAT das mudas de eucalipto. Botucatu-SP, 1993

\begin{tabular}{|c|c|c|c|c|c|c|c|c|}
\hline \multirow{3}{*}{ Tratamento } & \multicolumn{8}{|c|}{ Solução de Solo } \\
\hline & \multicolumn{8}{|c|}{$\%$} \\
\hline & \multicolumn{2}{|c|}{$\mathrm{N}$} & \multicolumn{2}{|c|}{$\mathrm{K}$} & \multicolumn{2}{|c|}{$\mathrm{Ca}$} & \multicolumn{2}{|c|}{$\mathrm{Mg}$} \\
\hline $1(0,0$ BRADC $+0,00$ Sphagnum $=0,000 \%$ C $)$ & \multicolumn{2}{|c|}{0,150} & \multicolumn{2}{|c|}{0,505} & \multicolumn{2}{|c|}{2,32} & \multicolumn{2}{|c|}{0,553} \\
\hline $2(0,5 \mathrm{BRADC}+0,00$ Sphagnum $=0,000 \% \mathrm{C})$ & \multicolumn{2}{|c|}{0,092} & \multicolumn{2}{|c|}{0,993} & \multicolumn{2}{|c|}{2,55} & \multicolumn{2}{|c|}{0,693} \\
\hline $3(1,0$ BRADC $+0,00$ Sphagnum $=0,000 \% \mathrm{C})$ & \multicolumn{2}{|c|}{0,156} & \multicolumn{2}{|c|}{0,726} & \multicolumn{2}{|c|}{2,17} & \multicolumn{2}{|c|}{0,496} \\
\hline $4(2,0$ BRADC $+0,00$ Sphagnum $=0,000 \% \mathrm{C})$ & \multicolumn{2}{|c|}{0,139} & \multicolumn{2}{|c|}{1,613} & \multicolumn{2}{|c|}{2,59} & \multicolumn{2}{|c|}{0,867} \\
\hline $5(3,0$ BRADC $+0,00$ Sphagnum $=1,299 \% \mathrm{C})$ & \multicolumn{2}{|c|}{0,236} & \multicolumn{2}{|c|}{2,626} & \multicolumn{2}{|c|}{3,04} & \multicolumn{2}{|c|}{1,323} \\
\hline $6(0,0$ BRADC $+4,15$ Sphagnum $=1,299 \%$ C $)$ & \multicolumn{2}{|c|}{0,673} & \multicolumn{2}{|c|}{1,440} & \multicolumn{2}{|c|}{3,19} & \multicolumn{2}{|c|}{1,093} \\
\hline $7(0,5$ BRADC $+3,46$ Sphagnum $=1,299 \% \mathrm{C})$ & & & & 53 & 2, & & & \\
\hline $8(1,0$ BRADC $+2,77$ Sphagnum $=1,299 \%$ C $)$ & & & & 10 & 2, & & & \\
\hline $9(2,0$ BRADC $+1,38$ Sphagnum $=1,299 \%$ C $)$ & & & & 70 & 2 , & & & \\
\hline Contrastes entre Tratamentos & $\mathrm{F}$ & $\operatorname{Pr}>\mathrm{F}^{1 /}$ & $\mathrm{F}$ & $\operatorname{Pr}>\mathrm{F}^{1 /}$ & $\mathrm{F}$ & $\operatorname{Pr}>\mathrm{F}^{1 /}$ & $\mathrm{F}$ & $\operatorname{Pr}>\mathrm{F}^{1 /}$ \\
\hline 1 vs. 2 & 0,12 & 73,72 & $2,82 *$ & 10,95 & 0,39 & 53,56 & 0,37 & 55,20 \\
\hline 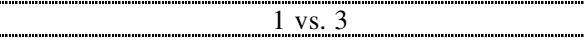 & 0,00 & 96,85 & 0,58 & 45,54 & 0,18 & 67,59 & 0,06 & 80,91 \\
\hline $\begin{array}{ll}1 \text { vs. } 4 \\
1 \text { van }\end{array}$ & 0,01 & 93,70 & $14,52^{*}$ & 0,12 & 0,54 & 46,92 & 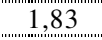 & 19,09 \\
\hline 1 vs. 5 & 0,64 & 43,27 & $53,21 *$ & 0,01 & $4,01 *$ & 5,91 & $11,07 *$ & 0,34 \\
\hline 6 vs. 7 & $5,77 *$ & 2,61 & 0,51 & 48,18 & 2,07 & 16,57 & 1,64 & 21,45 \\
\hline 6 vs. 8 & $0,13 *$ & 0,47 & 2,73 & 11,48 & $8,71^{*}$ & 0,79 & $7,02 *$ & 1,54 \\
\hline 6 vs. 9 & $10,0^{*}$ & 0,49 & 0,01 & 90,94 & $7,40^{*}$ & 1,32 & $4,54 *$ & 4,56 \\
\hline Sem Sphagnum vs. Com Sphagnum & $4,42 *$ & 4,85 & $6,20^{*}$ & 2,22 & 0,62 & 43,96 & 0,60 & 44,58 \\
\hline 1 vs. 6 & $9,88^{*}$ & 0,51 & $10,33^{*}$ & 0,46 & $5,80^{*}$ & 2,58 & $5,44 *$ & 3,02 \\
\hline 2 vs. 7 & 1,17 & 29,26 & 1,00 & 33,02 & 0,12 & 73,65 & 0,20 & 66,00 \\
\hline 3 vs. 8 & 0,01 & 93,70 & 1,19 & 28,97 & 0,01 & 90,58 & 0,01 & 94,33 \\
\hline 4 vs. 9 & 0,00 & 95,27 & 0,30 & 58,81 & 1,11 & 30,56 & 1,33 & 26,28 \\
\hline 6 vs. 5 & $5,48^{*}$ & 2,96 & $20,81^{*}$ & 0,02 & 0,16 & 68,92 & 0,99 & 33,22 \\
\hline $\mathrm{F}$ tratamentos & 2,10 & 7,98 & 10,03 & 0,01 & 2,05 & 8,74 & 2,80 & 2,65 \\
\hline $\mathrm{CV}(\%)$ & 92 & & & 17 & 17 & 54 & & \\
\hline
\end{tabular}

${ }^{1 /}$ Probabilidade com significância em porcentagem de 0,01 a 100; as médias com * são significativas a 10\% de probabilidade.

Trabalhos posteriores deverão ser conduzidos, procurando isolar e identificar possíveis compostos alelopáticos presentes em B. decumbens. Após a identificação destes, estudos podem ser feitos sobre genética, especificidade, influências e conseqüências ao meio ambiente.

Nas condições em que o presente trabalho foi realizado, pode-se concluir que as matérias secas de todas as plantas daninhas testadas inibiram o desenvolvimento das mudas de Eucalyptus grandis; a matéria seca de A. conyzoides e $B$. decumbens nas proporções máximas $(3 \% \mathrm{p} / \mathrm{p})$ reduziu, de forma mais acentuada, todas as variáveis estudadas nas plantas de eucalipto; as mínimas proporções de $B$. decumbens, incorporadas ao solo, que apresentaram efeito inibitório foram de 0,5 e $1 \%$, com e sem Sphagnum sp., respectivamente; a altura, o teor de clorofila, a área foliar e a matéria seca de folhas, caule e raiz foram as características da cultura mais sensiveis aos efeitos dos tratamentos; as análises da solução de solo indicam que não ocorreram grandes modificações do potencial osmótico, condutividade elétrica, $\mathrm{pH}$ e teores de macronutrientes desta.

\section{LITERATURA CITADA}

ALSAADAWI, I. S.; SAKERI, F. A. K.; AL-DULAIMY, S. M. Allelopathic inhibition of Cynodon dactylon (L.) Pers and others plant species by Euphorbia prostrata (L.). J. Chem. Ecol., v. 16, p. 2747-54, 1990.

ALVES, P. L. C. A. Interações alelopáticas entre plantas daninhas e hortaliças. In: SIMPÓSIO NACIONAL SOBRE MANEJO INTEGRADO DE PLANTAS DANINHAS EM HORTALIÇAS, 1992, Botucatu. Anais... Botucatu: UNESP, 1992. p. 19-43.

ANAYA, A. L. et al. Allelopathic pontencial of compounds isolated from Ipomoea tricolor (Convolvulaceae). J. Chem. Ecol., v. 16, p. 2145-52, 1990.

BHOWMIK, P. C.; DOLL, J. D. Corn and soybean response to allelopathic effects of weed and crop residues. Agron. J., v. 74, p. 601-606, 1982. 
BHOWMIK, P. C.; DOLL, J. D. Allelopathic effects of annual weed residues on growth and nutrient uptake of corn and soybeans. Agron. J., v. 76, p. 383-388, 1984.

BLUM, U.; WEED, S. B.; DALTON, B. R. Influence of various soil factors on the effects of ferulic acid on leaf expansin of cucumber seedlings. Plant Soil, v. 98, p. 111130, 1987.

CHOU, C. H. Allelopathy in sustainable Agriculture in Taiwan. In: ASIAN-PACIFIC WEED SCIENCE SOCIETY CONFERENCE TAIPEI, 11., 1987. China. Proceedings... China: 1987. p. 6.

CHOU, C. H.; LIN, H. J. Autointoxication mechanism of Oryza sativa. I. Phytotoxic effects of decomposing rice residues in paddy soil. J. Chem. Ecol., v. 2, p. 353-367, 1976.

CHOU, C. H. Allelopathic research of subtropical vegetation in Taiwan. IV. Comparative Phytotoxic nature of leachate from four subtropical grasses. J. Chem. Ecol., v. 15 , p. $2149-2159,1989$

CHOU, C. H.; KUO, Y. L. Allelopathic research in subtropical vegetation in Taiwan III. Allelopathic exclusion of understory species by Leucena leucocephala. J. Chem. Ecol., v. 12, p. 1431-1448, 1986.

DALTON B. R.; BLUM, U.; WEED, S. B. Allelopathic substances in ecosystems: effectiveness of sterile soil components in altering recovery of ferulic acid. J. Chem. Ecol., v. 9, p. 1185-1201, 1983.

DROST, D. C.; DOLL, D. Allelopathic effect of yellow nutsedge (C. esculentus) on corn (Zea mays) and soybeans (Glycine max). Weed Sci., v. 28, p. 229-233, 1980.

DROST, D. C.; DOLL, J. C.; MOODY, K. Effect of live plant leaching and different residue sources on the allelopathy of C. esculentus. Weed Sci., v. 7, p. 34-39, 1980.

DUBEY, P.; KHARE, N.; GUPTA, P. C. A new flavonoid glycoside from the leaves of Ipomoea fistulosa. Curr. Sci., v. 51, p. 351-352, 1982.

FUERST, E. P.; PUTNAN, A. R. Separating the competitive and allelopathic components of interference: theoretical principles. J. Chem. Ecol., v. 9, p. 937-944, 1983.

GLIESSMAN, S. R. Allelopathic interactions in crop-weed mixtures: applications for weed managment. J. Chem. Ecol, v. 9, p. 991-999, 1983.

HOSSNER, L. R.; PHILLIPS, D. P. Extraction of soil solution from flooded soil using a porous plastic filter. Soil Sci., v. 115 , n. 1, p. 87-88, 1973.

ITO, M.; KOBAYASHI, H; UEKI, K. Allelopathic potencial of Digitaria adscendens: Inhibitory effects of previously grown soil on growth and emergence. In: ASIAN-PACIFIC, WEED SCIENCE SOCIETY CONFERENCE TAIPEI, 11., 1987, China. Proceedings... China: 1987. p. 607-612.

Planta Daninha, Viçosa-MG, v.21, n.3, p.343-354, 2003
LABONTE, D. R.; DARDING, R. L. Noncompetitive effects of the morningglory on the growth of soybeans. Trans. N.Y. Acad. Sci., v. 81, p. 39-44, 1988.

LABRADA, R; FONT, C.; PAZOS, R. Allelopathy of perennial weeds on different crop plants: effect on germination. Cienc. Tec. Agric., v. 9, p. 71-83, 1986.

LORENZI, H. Plantas daninhas do Brasil. 2.ed. Nova Odessa: 1991. $440 \mathrm{p}$.

MORAES, J. F. V.; DYNIA, J. F. Uso de cápsulas porosas para extrair solução do solo. Pesq. Agropec. Bras., v. 25, n. 10 , p. 1523-1528, 1990.

NEPTUNE, A. M. L. Curso de nutrição de plantas, fertilidade do solo, adubos e adubação. Piracicaba: ESALQ-USP, 1976. v.1. 240 p.

NORSTAT, F. A.; McCALLA, T. M. Phytotoxic substance from a species of Penicillium. Science, v. 140, p. 410-411, 1963.

PUTNAM, A. R.; TANG, C. S. The science of allelopathy. New York: John Wiley \& Sons, 1986. 317 p.

RAIJ, B. V.; QUAGGIO, J. A. Método de análise de solo para fins de fertilidade. B. Tec. Inst. Agron., n. 81, p. 1-31, 1983.

RICE, E. L. Allelopathy. New York: Academic Press, 1974. $353 \mathrm{p}$.

RICE, E. L. Allelopathy an update. Bot. Rev., v. 45, p. 15109, 1979.

RICE, E. L. Overview of Allelopathy. In: NATIONAL SYMPOSIUM “ALLELOPATHY IN AGROECOSYSTEMS”, 1., 1992, Hisar. Proceedings... Hisar: Indian Society of Allelopathy/Haryana Agricultural University, 1992. p. 1.

SARRUGE J. R.; HAAG, H. P. Análise química em plantas. Piracicaba, Escola Superior de agricultura "Luiz de Queiroz", 1974. 56 p.

SCHOW, M. K.; EINHELLIG, F. A. Allelopathic effects of cultivated sunflower on grain sorghum. Bot. Gaz., v. 143, p. 505-510, 1982.

STEVENS, G. A.; TANG, C. S. Inhibition of crop seedling growth by hydrophobic root exudates of the weed Bidens pilosa. J. Trop. Ecol., v. 3, p. 91-94, 1987.

TABER, W. A.; VINING, L. C. Clavine and lysergic acid alkaloids in varieties of morningglory. Phytochemstry, v. 2, p. 65-70, 1963.

WANG T. S. C.; LI, S. W.; FERNG, Y. L. Catallytic pdymerrization of phenolic compounds by clay minerals. Soil Sci., v. 126, p. 15-21, 1978.

WILSON, R. E.; RICE, E. L. Allelopathy as expressed by Helianthus annuus and its role in old field succession. Bull. Torrey Bot. Club, v. 95, p. 432-448, 1968. 